\title{
Negritude no Brasil: Publicações em análise do comportamento
}

\author{
Pedro Henrique Jardim e Paula Suzana Gioia \\ Programa de Estudos Pós-Graduados em Psicologia Experimental: Análise do Comportamento, Pontifícia \\ Universidade Católica de São Paulo (PUC-SP)
}

Submissão: 15 jan. 2020.

Aceite: 13 ago. 2021.

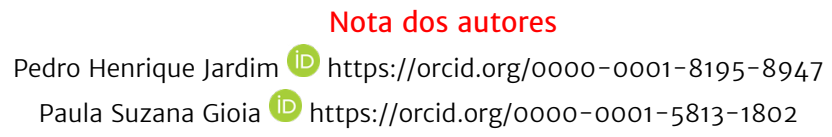

Correspondências referentes a este artigo devem ser enviadas para Pedro Henrique Jardim, Rua Artur Ferreira de Abreu, 344, Jardim Guaraú, Butantã, São Paulo, SP, Brasil. CEP 05547-150. E-mail: pedrohjpsi@gmail.com 


\section{Resumo}

Dados quantitativos revelam diferenças entre negros e não negros em relação à violência no Brasil, e esse fato abre espaço para discutir o racismo. Autores apontam que há necessidade de levar em consideração aspectos históricos, como o escravismo, para compreender a situação atual do fenômeno. Este estudo teve como objetivo discutir a contribuição da Análise do Comportamento, por meio de seus estudos, para a construção de comportamentos não racistas. Realizou-se busca nos Periódicos Capes, e selecionaram-se estudos produzidos nos últimos 20 anos (2000-2020) que contivessem as palavras de busca racism, racial e Behavior Analysis. Os estudos discutiam sobre ferramentas para mensuração de viés racial implícito e também formas de intervenção. Conclui-se que a Análise do Comportamento, embora seja uma ciência eficaz para mudança de comportamento, ainda precisa aumentar sua produção sobre o racismo.

Palavras-chave: racismo, racismo estrutural, análise do comportamento, equivalência de estímulos, viés racial implícito

\section{BLACKNESS IN BRAZIL: PUBLICATIONS IN BEHAVIOR ANALYSIS}

\section{Abstract}

Quantitative data has been revealing differences between blacks and non-blacks regarding violence in Brazil. This fact opens space to discuss racism. Authors has been pointing out that there is a need to consider historical aspects, such as slavery, to understand the current stage of the phenomenon. This study aimed to discuss the contribution of Behavior Analysis, through its studies, to the construction of non-racist behaviors. Having the last 20 years as time frame (2000-2020), a search and a selection of studies that contained the keywords racism, racial, and Behavior Analysis were carried out in the Capes Journals database, from Brazil. The found studies discussed tools for measuring implicit racial bias as well as ways of intervention. It was concluded that Behavior Analysis, although an effective science for behavior change, still needs to increase its production on racism.

Keywords: racism, structural racism, behavior analysis, stimulus equivalence, implicit racial bias

\section{NEGRITUD EN BRASIL: PUBLICACIONES EN ANÁLISIS DEL COMPORTAMIENTO}

\section{Resumen}

Datos cuantitativos han revelado diferencias entre negros y no negros con respecto a la violencia en Brasil. Esta realidad abre espacio para discutir el racismo. Autores vienen señalando que es necesario considerar aspectos históricos, como la esclavitud, para comprender el escenario actual del fenómeno. Este estudio tuvo como objetivo discutir la contribución del Análisis del Comportamiento, a través de sus pesquisas, a la construcción de conductas no racistas. Se realizaron una búsqueda y selección de 
estudios que contenían las palabras clave racism, racial y Behavior Analysis, en la base de datos Periódicos Capes, de Brasil, escritos en los últimos 20 años (2000-2020). Los estudios encontrados discutieron herramientas para medir el sesgo racial implícito, así como formas de intervención. Se llegó a la conclusión de que el Análisis del Comportamiento, aunque es una ciencia eficaz para el cambio de conductas, todavía necesita aumentar su producción sobre el racismo.

Palabras clave: racismo, racismo estructural, análisis del comportamiento, equivalencia de estímulos, sesgo racial implícito 
Dados apontados pelo Atlas da violência: 2019, do Instituto de Pesquisa Econômica Aplicada - Ipea (2020), revelam que, no Brasil, em 2018, 75,7\% das pessoas vítimas de homicídios eram classificadas como negras (pretas e pardas). Entre 2008 e 2018, houve um aumento de $11,5 \%$ na taxa de homicídios para negros. Em contrapartida, para não negros, houve uma diminuição de $12,9 \%$. Já em relação à violência policial, dados extraídos da $14^{\mathrm{a}}$ edição do Anuário brasileiro de segurança pública, elaborado pelo Fórum Brasileiro de Segurança Pública (FBSP), publicado em 2020, revelam que $79,1 \%$ das pessoas mortas em intervenções policiais, em 2019, eram negras. Destaca-se que, no primeiro semestre de 2020, o número de mortes por intervenção policial aumentou $6 \%$. Esses dados apontam para a questão discutida por Mercer (2020): a polícia mata mais negros do que qualquer outra raça.

Uma necessária compreensão da questão negra no Brasil deve iniciar-se com o período escravagista. Moura (2014), com sua vasta contribuição para a compreensão do racismo estrutural, denomina escravismo pleno o período entre o século XVI (início do tráfico internacional de africanos escravizados) e a Lei Eusébio de Queiroz (fim do tráfico internacional de africanos escravizados), em 1850, pois o comércio escravista dominava as dinâmicas social, política e econômica quase inteiramente. As relações interpessoais nesse período, portanto, tinham como essência o modo de produção entre duas classes vigentes: escravizados e senhores.

Da mesma forma que Moura (2014), Almeida (2019) destaca esse período como marco histórico para a ideia e prática do racismo como uma estrutura social no Brasil - e complementa sua análise ao pontuar o aspecto filosófico do escravismo pleno, que ocorreu no período do lluminismo, no qual o ser humano não era apenas sujeito, mas também objeto de conhecimento. Portanto, nesse período, pessoas de diferentes grupos sociais eram classificadas com base em suas características físicas e culturais: o europeu era o modelo, e havia diferenciação entre civilizado e selvagem e, posteriormente, entre civilizado e primitivo: quanto mais distante do padrão europeu, mais "primitiva" era a pessoa. Dessa forma, segundo o autor, o uso da raça, além de um mecanismo para classificar pessoas, também justificava o coIonialismo por parte do povo europeu em relação aos demais povos. Nas palavras de Almeida (2019), com o "uso da razão", os europeus foram capazes de diversos genocídios, como as ações contra os povos da América e África:

O iluminismo tornou-se o fundamento filosófico das grandes revoluções liberais que, a pretexto de instituir a liberdade e livrar o mundo das trevas e preconceitos da religião, iria travar guerras contra as instituições absolutistas e o poder tradicional da nobreza. As revoluções inglesas, americana e francesa foram o ápice de um processo de reorganização do mundo, de uma longa e brutal transição das sociedades feudais para a sociedade capitalista em que a composição filosófica do homem universal, dos direitos universais e da razão universal mos- 
trou-se fundamental para a vitória da civilização. Esta mesma civilização que, no século seguinte, seria levada para outros lugares do mundo, para os primitivos, para aqueles que ainda não conheciam os benefícios da liberdade, da igualdade, do Estado de direito e do mercado. E foi esse movimento de levar a civilização para onde ela não existia que redundou em um processo de destruição e morte, de espoliação e aviltamento, feito em nome da razão e a que se denominou colonialismo.

Seguindo o mesmo enredo citado por Almeida (2019) e Moura (2014), em meados do século XIX, surge a ideologia racial, entendida como política de distinções raciais e seus papéis na sociedade. Nesse período, os europeus precisavam justificar a exploração de determinados povos, como africanos e asiáticos, considerando-os "bárbaros" e "primitivos", enquanto viam a si próprios como "civilizadores" (Oliveira, 2008). Para sustentar essas crenças, foram utilizados pressupostos biológicos baseados no positivismo e na interpretação equivocada de teorias das ciências naturais, como o darwinismo social e a teoria de Mendel, também utilizadas como justificativas para impedir a miscigenação (Oliveira, 2008; Romero, 2015), já que negros eram vistos como pessoas imorais, lascivas e violentas (Almeida, 2019). São estereótipos que parecem ainda presentes, como aponta Mercer (2020), ao expor a violência policial sofrida por pessoas negras atualmente.

Quando surgiu, no Brasil, em 1918, a Sociedade Eugênica de São Paulo defendia a necessidade de uma direção científica e de a nação ser planejada por meio de parâmetros raciais, a fim de propiciar progresso ao país. Para isso, seria necessário solucionar o "problema racial" do Brasil e encontrar um papel para negros e mestiços (Romero, 2015). Para os eugenistas, segundo Romero (2015), não havia necessidade de impedir a hibridação, mas de "injetar" sangue branco na população, ao cruzar mestiços com europeus. Dessa forma, após a quarta ou quinta geração, ter-se-ia uma população branca. A miscigenação, portanto, aparecia no discurso nacionalista como uma forma de "cura social" e perpetuava uma hierarquia racial, segundo a qual quanto mais próximo ao branco, melhor; e, quanto mais próximo ao negro, pior, tanto por fatores biológicos quanto por práticas culturais.

É compreensível, portanto, porque para Almeida (2019) o entendimento do racismo esbarra na relação de poder estabelecida entre grupos, em que um grupo tem o domínio sobre o outro por meio das instituições. Ao tratar dos aspectos institucionais do racismo, o autor destaca que as instituições reproduzem o racismo e estruturam relações políticas, econômicas, jurídicas e familiares.

\section{A Análise do Comportamento e a compreensão do racismo}

Tendo em vista os aspectos históricos apresentados e a situação atual do negro no Brasil, percebe-se a necessidade de propostas científicas que mudem essa realidade social, e a 
Análise do Comportamento pode ser uma das ciências capazes de contribuir para explicar o que mantém o fenômeno e para criar mecanismos de mudanças.

Em uma visão analítico-comportamental, é necessário levar em consideração que qualquer comportamento operante foi instalado via reforçamento, e as instituições podem ser vistas, segundo Skinner (1953/2003), como agências de controle responsáveis por organizar contingências por meio de regras para seus membros. Diferentemente do que Almeida (2019) discorre sobre as instituições, as agências de controle, na visão analítico-comportamental, produzem e mantêm contingências sociais, e, dessa forma, a descrição de regras mais equalitárias também poderia se formulada por meio de novas leis e sanções advindas para o não cumprimento delas.

A relação de poder, descrita por Almeida (2019), poderia ser entendida por um analista do comportamento pelo controle social exercido por um grupo econômico. Para Skinner (1953/2003), o controle está sempre presente, e todo comportamento é passível de controle, seja identificado ou não, incluindo as relações sociais. No entanto, ao que parece, a relação de poder está relacionada às situações de controle aversivo vivida pela população negra em comparação à população branca, somada ao fato de as agências de controle promoverem regras nas quais brancos são beneficiados em detrimento de negros, fato observado em todas as agências de controle citadas por Skinner (1953/2003) - governo, religião, cultura e educação.

A Análise do Comportamento não identifica a raça como uma variável de controle relevante para a explicação do comportamento. No entanto, é publicado, em 1994, o livro A curva do sino, de Richard Herrnstein e Charles Murray, no qual é retomada a ideologia da inferioridade do negro, apoiada no quociente de inteligência (QI). Segundo os autores, de acordo com resultados de testes de inteligência, negros têm QIs inferiores a brancos. No entanto, é relevante destacar que tais testes parecem desconsiderar a trajetória social de opressão, negligência e desigualdade na história de vida dos negros - trajetória apontada por Moura (2014, p. 35, grifos do autor) como fundamento para a compreensão racial:

Chamamos de escravismo pleno àquele período de escravidão que se estende no Brasil do ano de 1550 (+-) até aproximadamente 1850, quando é jurídica e efetivamente extinto o tráfico internacional de escravos africanos. Abrange, portanto, todo o período colonial, a fase do reinado de D. João VI, o império de D. Pedro I e D. Pedro II. Nesse longo período de mais de trezentos anos, estrutura-se e dinamiza-se o modo de produção escravista no Brasil com todas as características que determinarão o comportamento básico das duas classes fundamentais da sua estrutura social: senhores e escravos.

A curva do sino recebeu críticas por parte da comunidade de analistas do comportamento devido ao ideal eurocêntrico e racista. Em uma dessas críticas, Andery e Sério (1997) 
analisaram as afirmações contidas no livro por meio de uma visão baseada na filosofia do behaviorismo radical - e demonstraram a existência de fatores metodológicos e éticos errôneos, como a mensuração do que se chama "inteligência".

Racismo trata de preconceito, discriminação ou comportamento hostil direcionados a pessoas de raça ou etnia diferente da dominante. Sistemas racistas afirmam uma hierarquia entre raças/etnias, na qual uma é exaltada em detrimento das demais, e podem preconizar o isolamento das outras raças/etnias ou até mesmo seu extermínio (Houaiss, 2001). Skinner (1953/2003, p. 325) define comportamento social como "o comportamento de duas ou mais pessoas em relação a uma outra ou, em conjunto, em relação ao ambiente comum". Portanto, é possível dizer que ocorrem, no caso, sistemas comportamentais entrelaçados. Dessa forma, a primeira consideração a ser feita é compreender o racismo como um comportamento social: uma contingência operante em que uma pessoa tem função de estímulo discriminativo e/ou de consequência (reforçadora ou aversiva) para outra.

Uma tentativa de definição comportamental e operante de racismo apoiada em Sidman (1989/1995) leva à possível conclusão de que se trata de uma classe de respostas identificada como racista que afeta e é afetada pelo meio. As contingências seriam aversivas e aplicadas a um determinado grupo socialmente hostilizado, visando mantê-lo em posição submissa, com reforçador generalizado para quem as aplica. Esse tipo de controle geraria respostas de fuga e esquiva por parte de quem é submetido e respostas de contracontrole dirigidas ao grupo controlador. Supõe-se, ainda, que, pelo fato de as repostas racistas não serem inatas, foram aprendidas e transmitidas ao longo dos anos dentro de um grupo social, construídas a partir de relações sociais e verbais estabelecidas em uma comunidade verbal.

Uma análise também possível do racismo trazida pelos analistas do comportamento é entendê-lo como prática cultural. Uma prática cultural é a repetição ou manutenção de ações ao longo das gerações ou da mesma geração: mesmo após a substituição de seus membros, um determinado grupo continua a comportar-se de forma semelhante ao anterior. É cabível, portanto, dizer que membros antigos ensinam aos novos membros como devem agir. Trata-se da propagação de comportamentos similares em que um indivíduo afeta outro indivíduo, de modo a produzir um comportamento semelhante ao seu ou ao de outrem, e a pessoa afetada posteriormente tende a afetar outros indivíduos, propagando o mesmo comportamento (Sampaio \& Andery, 2010).

Para explorar a transmissão do racismo, é necessário focar como essa prática é aprendida, levando-se em conta os modelos explicativos da Análise do Comportamento. Dessa maneira, o racismo pode ser absorvido por dois tipos de aprendizagem: respondente e operante. A aprendizagem respondente pode ocorrer quando há pareamento de estímulos: uma palavra pareada com um estímulo aversivo, por exemplo, pode exercer função de estímulo condicionado e eliciar respostas semelhantes às eliciadas pelo estímulo original. Assim, por 
exemplo, palavras como negro, apresentadas em situações aversivas, como as que envolvem medo, desgosto ou aversão, podem, por si sós, gerar reações emocionais semelhantes quando apresentadas sozinhas em situações futuras (Arhin \& Thyer, 2004).

Por sua vez, a aprendizagem operante por imitação pode estar presente na aquisição de algumas respostas racistas, principalmente no repertório de crianças e jovens. Caso uma criança veja constantemente seus pais emitirem essas respostas, tem chances de repetir o comportamento pela alta probabilidade de ser reforçada da mesma forma que seus pais. A aprendizagem operante por reforçamento positivo pode ocorrer quando uma criança recebe aprovação de seu grupo social ao ofender uma pessoa negra. Dessa forma, ela tenderá a repetir, em outro momento, respostas semelhantes diante de pessoas negras. Já na aprendizagem por reforçamento negativo, na qual há um aumento da frequência da resposta por meio da eliminação de um estímulo aversivo, uma pessoa tenderá a esquivar-se, no futuro, de emitir respostas que foram punidas, como ouvir música de pessoas negras, e, como consequência, receber chacotas de amigos ou conhecidos (Arhin \& Thyer, 2004).

Respostas racistas, finalmente, podem emergir de algumas poucas relações entre estímulos não diretamente ensinadas (equivalência de estímulos): se, por exemplo, são aprendidas as relações negro-pobre e pobre-ladrão, é provável que emerja a relação negro-ladrão, mesmo que ela não tenha sido diretamente ensinada (Rose, 1993).

Considerando os aspectos apresentados sobre racismo e a inserção da Análise do Comportamento na discussão do fenômeno, o objetivo deste estudo foi discutir a possível contribuição da Análise do Comportamento, por meio de seus estudos aplicados, para a construção de comportamentos não racistas.

\section{Seleção dos manuscritos}

Os documentos que serviram para atender ao objetivo deste estudo foram selecionados na biblioteca digital Periódicos da Coordenação de Aperfeiçoamento de Pessoal de Nível Superior (Capes), que inclui bases de dados relevantes para a psicologia, como a PsycINFO e a BVS-Psi. Utilizaram-se, para a seleção de artigos, as palavras-chave racism, racial e Behavior Analysis, que dizem respeito ao foco da discussão pretendida.

$O$ procedimento envolveu duas buscas. A primeira ocorreu com racism, contida em qualquer campo; o operador booleano AND; e Behavior Analysis, exata e em qualquer campo. A segunda busca ocorreu com racial, contida no título; o operador booleano AND; e Behavior Analysis, exata e em qualquer campo. Foram selecionados os artigos, após a leitura dos resumos, em um período dos últimos 20 anos (2000-2020).

Como critério de inclusão, foram selecionados: artigos em português ou inglês; teórico-conceituais, de revisão ou pesquisas experimentais aplicadas; escritos por analistas do comportamento; os conteúdos estavam relacionados diretamente ao racismo ou discriminação 
racial contra pessoas negras; e todos os artigos foram publicados em periódicos revisados por pares.

Como critério de exclusão, não foram selecionados artigos em que a raça negra não era investigada sob a ótica da discriminação racial (por exemplo, relacionamentos homoafetivos entre afro-americanos; afro-americanos e hipertensão).

A busca resultou em cinco artigos. Um pesquisador independente realizou, então, a busca na mesma base de dados e com as mesmas palavras-chave das duas buscas, o que resultou no índice de integridade do procedimento de $80 \%$, já que o pesquisador identificou quatro dos cincos artigos encontrados.

\section{O que a Análise do Comportamento tem a oferecer para a compreensão do racismo dirigido a negros?}

A exclusão de muitos artigos indicou que as relações étnico-raciais são estudadas principalmente por ciências relacionadas às áreas sociais e políticas. Quanto à Análise do Comportamento, embora existam diferentes técnicas e campos de atuação, esse tema parece pouco estudado em relação a outros, como comportamento verbal e desenvolvimento atípico.

Os artigos "Combining the implicit relational assessment procedure and the recording of event related potentials in the analysis of racial bias: A preliminary study" e "Exploring racial bias in a European country with a recent history of immigration of black Africans" foram escritos pelos mesmos autores (Power et al., 2017a, 2017b), e, em ambos os estudos, avaliou-se a eficácia da mensuração do viés implícito por meio de um software denominado Implicit Relational Assessment Procedure (IRAP). A expressão viés implícito traz a concepção de que existem respostas que, se abertas, podem ser punidas; portanto, uma pessoa evitaria emiti-las, o que, porém, não significa que o indivíduo não emita essas respostas de forma encoberta. Em outras palavras, as respostas racistas podem ser divididas em: 1 . viés racial implícito (encobertas), como pensamentos, preferências de escolhas e assim por diante; e 2. viés racial explícito (abertas), como respostas verbais e agressões físicas.

Vale ressaltar que viés implícito inclui também as respostas que a pessoa não tem intenção de emitir, não para evitar a punição, mas por não saber as contingências sob controle das quais está; por exemplo, dizer que as pessoas são todas iguais, mas, ao perceber uma pessoa negra, sentir-se desconfortável e não saber o motivo - no caso, uma possível forma de aprendizagem respondente (Arhin \& Thyer, 2004). Além disso, parece haver uma relação direta entre viés racial implícito e racismo estrutural, pois, como apontam Moura (2014) e Almeida (2019), as relações raciais tomaram conta das relações interpessoais. Portanto, as pessoas aprendiam a forma como deviam comportar-se, e esses comportamentos, ainda hoje, perpetuam essas relações. Dessa maneira, pode-se dizer que, enquanto o racismo se mantiver como estrutura, as pessoas tenderão a permanecer enviesadas. 
Quanto ao software utilizado, trata-se do IRAP. A partir dele, é possível mensurar algumas relações, nas quais os participantes tendem a não conseguir manipular o resultado. Então, avalia-se a consistência da relação: quanto mais rápida a pessoa responder, mais consistente é considerada a relação entre os estímulos, e vice-versa. Dessa maneira, apresentam-se a imagem de uma pessoa negra e um adjetivo positivo; em um segundo momento, apresentam-se a imagem de uma pessoa branca e um adjetivo negativo. Esses estímulos depois se alternam: a pessoa negra também é apresentada com adjetivos negativos, e a branca aparece ao lado de adjetivos positivos. É fornecida uma regra aos participantes, como clicar em sim quando a relação for negro/positivo e clicar em não quando for branco/positivo, e vice-versa. Considera-se uma relação mais consistente quando a pessoa responde com maior agilidade para uma relação do que para outra. No caso, o viés racial negativo para pessoas negras é identificado quando se responde mais rapidamente para a relação negro/negativo do que branco/negativo, ou, então, mais rapidamente para negro/negativo do que para branco/ negativo.

Em ambos os estudos (Power et al., 2017a, 2017b), foram apresentados resultados relevantes em relação ao IRAP, e isso sugere que o software é um mecanismo eficaz para esse tipo de mensuração. Para a Análise do Comportamento, também pode ser considerado um instrumento relevante na área de controle de estímulos, por permitir avaliar alguma propriedade do estímulo relacionada a outras propriedades de outros estímulos, sempre levando em consideração as características pré-experimentais obtidas com o participante. $O$ fato de o instrumento medir o tempo de latência da resposta contribui para que o participante tenha menor controle sobre os resultados. Parece, portanto, que, para analistas do comportamento interessados em investigar o racismo na sociedade, a utilização do IRAP é bastante pertinente como ferramenta de mensuração de mudança.

A utilização desse instrumento por analistas do comportamento foi confirmada pela busca de artigos realizada que permitiu encontrar dois estudos que seguem essa modalidade de mudança. O primeiro estudo é de Carvalho e Rose (2014) e teve como objetivo verificar a mudança de viés entre homens negros e símbolos negativos em crianças de 7 a 10 anos de idade. Utilizaram-se como estímulos: figuras abstratas, fotos de homens negros, fotos de homens brancos, fotos de personagens de desenho animado, além dos símbolos positivo e negativo feitos com o polegar. O procedimento foi composto por linha de base, treino e teste, e iniciou-se com a aplicação da Escala de Diferencial Semântico para aferir como as crianças avaliavam negros e brancos.

Após a confirmação de viés negativo em relação a negros, isto é, foram selecionadas as crianças nas quais se identificou viés negativo em relação a negros, iniciou-se o teste das faces (figuras de homens, brancos e negros; e símbolos abstratos, relacionados ao gesto positivo ou negativo), a fim de avaliar (por meio da escolha da posição do polegar) as escolhas dos 
participantes ante a relação entre estímulos de rostos de negros ou de brancos e estímulos reforçadores ou aversivos.

Para a mudança do viés racial, utilizou-se um procedimento em que, inicialmente, foram ensinadas as relações entre os sinais do polegar para cima ou para baixo e figuras abstratas ( $A B)$. Em seguida, foi iniciado o treino da relação entre os estímulos: figuras abstratas e as faces de brancos e negros (BC). Por fim, realizou-se um treino misto das relações entre sinais do polegar e figuras abstratas; e das relações entre figuras abstratas e fotos de pessoas $(A B / B C)$. A última fase do estudo tinha o intuito de verificar se ocorreram mudanças com o treino, comparativamente à linha de base. Os resultados indicaram que, para a maior parte dos participantes, não houve alteração do viés, ou seja, não foi possível a formação de classes entre símbolos positivos e homens negros, o que sugere resistência à reversão, possivelmente porque as crianças voltavam diariamente para um meio que as fez aprender as relações enviesadas. Como apontado por Almeida (2019), as instituições reproduzem comportamentos racistas que são estruturais, e família e escola fazem parte dessas instituições. Dessa forma, talvez o ensino não pudesse ser suficiente para competir com o meio social. Outra possibilidade para explicar a resistência à reversão relaciona-se ao fato de os sinais positivo e negativo não possuírem o significado adequado - função reforçadora ou aversiva.

A mesma preocupação investigativa tiveram Mizael et al. (2016), que conduziram uma replicação sistemática do estudo de Carvalho e Rose (2014) e objetivaram verificar se o ensino de novas relações de equivalência seria capaz de reverter classes pré-experimentais em crianças com viés racial negativo em relação a pessoas negras. Dito de outra forma, os autores pretenderam verificar se o ensino por meio do emparelhamento de fotos de pessoas negras com símbolos positivos (reforçadores) podia reverter o viés inicial.

Diferentemente de Carvalho e Rose (2014), para a seleção de participantes, Mizael et al. (2016) utilizaram o Manequim de Autoavaliação (Self-Assessment Manikin - SAM), com o intuito de avaliar experiências afetivas diante de imagens. Os participantes recebiam folhas de sulfite com a imagem de uma pessoa branca ou negra na parte superior. Na parte inferior, havia cinco desenhos de bonecos que representavam fisionomias de feliz até triste, e a resposta exigida era colocar um " $X$ " abaixo do boneco que correspondesse ao sentimento do participante diante da imagem da pessoa. Foram selecionadas 13 crianças, de ambos os sexos com idades entre 8 e 10 anos, que pareavam as fisionomias de negros com bonecos de expressões tristes. Os participantes selecionados passavam para uma segunda fase de teste, denominada Teste AC3, na qual os estímulos-modelo eram imagens do polegar com gesto positivo ou negativo, e os estímulos-comparação eram imagens de pessoas brancas, negras ou estímulos abstratos. Os participantes deviam clicar na figura das pessoas que correspondesse ao gesto do polegar. Assim, obteve-se a linha de base dos participantes que apresentaram viés. Apenas três participantes eram negros, e os dez restantes eram brancos. Os participantes 
foram divididos em dois grupos. Um deles trabalhou com estímulos apresentados na mesma tela (MTS Simultâneo - SMTS), e o outro grupo, com atraso na apresentação dos estímulos de 2s (DMTS). Como estímulos, utilizaram-se quatro fotos de pessoas negras, quatro de pessoas brancas, uma de polegar positivo, uma de polegar negativo e três de estímulos abstratos.

O próximo passo do procedimento foi o pré-treino, com o objetivo de ensinar aos participantes como era o procedimento de MTS. Na fase de treino, distribuíram-se os 13 participantes nos dois grupos (SMTS e DMTS), e o procedimento foi semelhante ao de Carvalho e Rose (2014), ou seja, eram ensinadas as relações: $A B$, polegares e figuras abstratas; $B C$, figuras abstratas e pessoas negras; e $A B B C$, uma mistura das duas relações anteriores. Após cada treino, era realizado um teste de simetria (por exemplo, se fosse $A B$, o teste seria $B A$ ), e, após a fase de treino, fez-se o teste de equivalência. Esperava-se que, diante do polegar positivo (A1), seria escolhida a figura de pessoa negra (C1), e vice-versa (relação não treinada anteriormente). Por fim, para verificar se ocorreram mudanças no viés constatado na avaliação da linha de base, repetiram-se o Teste AC3 e o SAM, além do IRAP.

No estudo de Mizael et al. (2016), todos os participantes selecionados, que pareavam negros a símbolos negativos, mudaram suas respostas para negros e símbolos positivos, resultado que não havia ocorrido na pesquisa de Carvalho e Rose (2014). Embora os participantes tenham sido divididos em dois grupos de treino, os resultados foram bastante semelhantes nos dois grupos. Essa pesquisa mostrou-se de grande relevância para o estudo do racismo, pois, além de evidenciar uma variável implícita (reversão de classes pré-experimentais em crianças com viés racial negativo em relação a pessoas negras), difícil de ser mensurada por meio de outros procedimentos que não utilizem a equivalência de estímulos e o IRAP, indicou uma forma de mudar a relação socialmente construída entre estímulos negativos e pessoas negras. Esses aspectos sustentam a importância de extensão da aplicação da pesquisa a novos participantes para aumentar a generalidade dos resultados obtidos por Mizael et al. (2016). Outro ponto indicado nos resultados da pesquisa é a necessidade de investigar se haverá generalização para novos ambientes sociais, fora do setting experimental, e se eles se manteriam no decorrer do tempo.

Por fim, o último estudo selecionado - "Can behavior analysis help us understand and reduce racism? A review of the current literature" (do português, "A análise de comportamento pode nos ajudar a entender e reduzir o racismo? Uma revisão da literatura atual") (Matsuda et al., 2020) - é um artigo teórico cujos autores propõem maneiras para que membros da comunidade científica da Análise do Comportamento aprendam sobre o racismo de uma forma comportamental. Os autores traçam um caminho que se inicia com a explicação das diferenças entre racismo, preconceito e viés; fazem uma explicação sobre a forma de aprendizagem também baseados nas propostas de Arhin e Thyer (2004); partem para os mecanismos de mensuração de viés, como o IRAP, e suas formas de reduzi-lo utilizando os estudos de Carvalho e 
Rose (2014) e Mizael et al. (2016); e finalizam com estudos que apontam a terapia de aceitação e compromisso (acceptance and commitment therapy - ACT) em conjunto com mindfulness como formas para diminuir a dominância de regras sobre o comportamento-problema - no caso, as respostas racistas. Os autores afirmam que não encontraram na alternativa proposta no estudo uma possível ferramenta para a solução do racismo e mencionam que, para a solução, é importante que o analista do comportamento explore o maior leque de intervenções comportamentais, tanto as tradicionais quanto as contextuais, pois, dessa forma, aumentam- se as possibilidades de encontrar intervenções eficazes para a mudança de comportamento racista.

Esse artigo, por apresentar uma revisão, descreve diferentes aspectos das outras pesquisas apresentadas, no entanto parece ser o artigo que melhor explica o racismo dentro de uma ciência do comportamento - e da pesquisa básica. Os autores estavam empenhados em analisar conceitos da Análise do Comportamento sobre o tema racismo e preconceito, além de apresentarem as terapias comportamentais de terceira geração nessa discussão do fenômeno. O que se observa nesse estudo é que o procedimento de mudanças de comportamento racista parte do indivíduo para o todo; as intervenções explicitadas tendem a mudar a relação do participante para com o racismo e se espera que generalize para fora do contexto experimental.

Levando-se em considerações os aspectos das agências de controle descritas por Skinner (1953/2003), denominadas de instituições por Almeida (2019), pode-se dizer que uma intervenção no âmbito externo à experimentação dirija-se às formulações de políticas públicas, por meio de regras contingentes, mais especificamente normas sociais transcritas como leis e os comportamentos esperados. Dessa forma, poder-se-ia alcançar uma mudança social, em conjunto com a individual, que seria mais eficaz na alteração de padrões comportamentais racistas.

\section{Conclusão}

A Análise do Comportamento é uma ciência capaz de trazer possibilidade de mudanças de comportamentos sociais, ao prever e controlar variáveis relacionadas ao comportamento, e ainda que tenham sido poucos os estudos apresentados - o que retrata a falta de pesquisa na área -, pôde-se perceber neste estudo que a Análise do Comportamento permite intervir no fenômeno do racismo.

Segundo Mercer (2020), no ano de 2020, foram realizadas diversas manifestações contra a violência policial sofrida por pessoas negras, tendo como estopim a morte de George Floyd, assassinado por asfixia por um policial branco em Minnesota, nos Estados Unidos. Casos como o de Floyd não são isolados - e sua morte gerou protestos e o movimento Black Lives Matter (Vidas Negras Importam) em todo o mundo, mesmo em um período marcado pela pandemia da coronavirus disease 2019 (Covid-19), momento em que o distanciamento social se 
tornou medida primária para o controle da propagação do coronavírus da síndrome respiratória aguda grave 2 (severe acute respiratory syndrome coronavirus 2 - Sars-CoV-2), o que sugere a urgência desses protestos.

Fatos como esse denotam a relevância de abordar o tema do racismo e de intervir no fenômeno. No entanto, a contribuição da Análise do Comportamento, por meio de publicações, tem sido tão escassa que ainda não há condições de oferecer procedimentos eficazes para a mudança do comportamento racista. Há, portanto, a necessidade de novos estudos que envolvam maior número de participantes e que busquem as mais diferentes formas de intervenção. Além disso, considerando as pesquisas de viés racial, deve-se levar em conta o estabelecimento de generalização do comportamento do participante fora do contexto de pesquisa. 


\section{Referências}

Almeida, S. (2019). Racismo estrutural. Pólen.

Andery, M. A., \& Sério, T. M. (1997). The bell curve: What has radical behaviorism to say about it? Behavior and Social Issues, 7(1), 69-82. https://doi.org/10.5210/bsi.v7i1.302

Arhin, A., \& Thyer, B. A. (2004). The causes of racial prejudice: A behavior-analytic perspective. In J. L. Chin (Ed.), The psychology of prejudice and discrimination: Racism in America (pp. 1-19). Praeger Publishers/Greenwood Publishing Group.

Carvalho, M. P., \& de Rose, J. C. (2014). Understanding racial attitudes through the stimulus equivalence paradigm. The Psychological Record, 64(3), 527-536. https://doi.org/10.1007/s40732014-0049-4

De Rose, J. C. (1993). Classes de estímulos: Implicações para uma análise comportamental da cognição. Psicologia: Teoria e Pesquisa, 9(2), 283-303. https://periodicos.unb.br/index.php/revistaptp/article/view/17219

Fórum Brasileiro de Segurança Pública. (2020). Anuário brasileiro de segurança pública (14ed.). FBSP.

Herrnstein, R. J., \& Murray, C. (1994). The bell curve: The reshaping of American life by differences in intelligence. Free.

Houaiss, A. (2001). Racismo. In Dicionário Houaiss da Língua Portuguesa. Objetiva. Dicionário on-line.

Instituto de Pesquisa Econômica Aplicada. (2020). Atlas da violência: 2019. IPEA.

Matsuda, K., Garcia, Y. A., Catagnus, R., \& Brandt, J. A. (2020). Can behavior analysis help us understand and reduce racism? A review of the current literature. Behavior Analysis in Practice, 13, 336-347. https://doi.org/10.1007/s40617-020-00411-4

Mercer, J. A. (2020). \#BlackLivesMatter in Religious Education: The REA Statement on the Murder of George Floyd. Religious Education, 115(4), 379-383. https://doi.org/10.1080/00344087. 2020.1790724

Mizael, T. M., de Almeida, J. H., Silveira, C. C., \& de Rose, J. C. (2016). Changing racial bias by transfer of functions in equivalence classes. The Psychological Record, 66(3), 451-462. https://doi. org/10.1007/s40732-016-0185-0

Moura, C. (2014). Dialética radical do Brasil negro (2. ed). Fundação Maurício Grabois.

Oliveira, I. M. A. (2008). A ideologia do branqueamento na sociedade brasileira [Unpublished Didactic-Pedagogical Production]. Universidade Estadual do Norte do Paraná.

Power, P. M., Harte, C., Barnes-Holmes, D., \& Barnes-Holmes, Y. (2017a). Combining the implicit relational assessment procedure and the recording of event related potentials in the analysis of racial bias: A preliminary study. The Psychological Record, 67(4), 499-506. https://doi. org/10.1007/s40732-017-0252-1

Power, P. M., Harte, C., Barnes-Holmes, D., \& Barnes-Holmes, Y. (2017b). Exploring racial bias in a European country with a recent history of immigration of Black Africans. The Psychological Record, 67(3), 365-375. https://doi.org/10.1007/s40732-017-0223-6

Romero, M. (2015). Ciência, construção da nação e exclusão social: São Paulo - Brasil (1889-1930). Le Cahiers ALHIM - Amérique Latine Histoire et Mémoire, 29. https://doi.org/10.4000/alhim.5258 
Sampaio, A. A. S., \& Andery, M. A. P. A. (2010). Comportamento social, produção agregada e prática cultural: Uma análise comportamental de fenômenos sociais. Psicologia: Teoria e Pesquisa, 26, 183-192. https://doi.org/10.1590/S0102-37722010000100020

Sidman, M. (1995). Coerção e suas implicações (M. A. Andery \& T. M. Sério, Trads.). Editorial Psy II.

Skinner, B. F. (2003). Ciência e comportamento humano (J. C. Todorov \& R. Azzi, Trads.; 11. ed.). Martins Fontes.

\section{EQUIPE EDITORIAL \\ Editora-chefe}

Cristiane Silvestre de Paula

\section{Editores associados}

Alessandra Gotuzo Seabra Ana Alexandra Caldas Osório Luiz Renato Rodrigues Carreiro Maria Cristina Triguero Veloz Teixeira

\section{Editores de seção} "Avaliação Psicológica"

Alexandre Serpa

André Luiz de Carvalho Braule Pinto

Luiz Renato Rodrigues Carreiro Vera Lúcia Esteves Mateus

"Psicologia e Educação"

Alessandra Gotuzo Seabra

Carlo Schmidt

Regina Basso Zanon
"Psicologia Social e Saúde das Populações"

Enzo Banti Bissoli

Marina Xavier Carpena

\section{"Psicologia Clínica"}

Carolina Andrea Ziebold Jorquera

Julia Garcia Durand

Natalia Becker

"Desenvolvimento Humano"

Maria Cristina Triguero

Veloz Teixeira

Rosane Lowenthal

Suporte técnico

Maria Fernanda Liuti

Bento da Silva

Camila Fragoso Ribeiro
PRODUÇÃO EDITORIAL

Coordenação editorial

Ana Claudia de Mauro

\section{Estagiários editoriais}

Júlia Lins Reis

Élcio Carvalho

Pietro Menezes

Preparação de originais

Carlos Villarruel

\section{Revisão}

Vera Ayres

\section{Diagramação}

Acqua Estúdio Gráfico 